\title{
BINCOR: An R package for Estimating the Correlation between Two Unevenly Spaced Time Series
}

by Josue M. Polanco-Martinez, Martin A. Medina-Elizalde, Maria Fernanda Sanchez Goni, Manfred Mudelsee

\begin{abstract}
This paper presents a computational program named BINCOR (BINned CORrelation) for estimating the correlation between two unevenly spaced time series. This program is also applicable to the situation of two evenly spaced time series not on the same time grid. BINCOR is based on a novel estimation approach proposed by Mudelsee (2010) for estimating the correlation between two climate time series with different timescales. The idea is that autocorrelation (e.g. an AR1 process) means that memory enables values obtained on different time points to be correlated. Binned correlation is performed by resampling the time series under study into time bins on a regular grid, assigning the mean values of the variable under scrutiny within those bins. We present two examples of our BINCOR package with real data: instrumental and paleoclimatic time series. In both applications BINCOR works properly in detecting well-established relationships between the climate records compared.
\end{abstract}

\section{Introduction}

There are several approaches for quantifying the potential association between two evenly spaced climate time series, e.g. Pearson's and Spearman's correlation or the cross-correlation function (CCF). However, these methods should not be directly applied when the time series are unevenly spaced ("irregular"), particularly when two time series under analysis are not sampled at identical points in time, as is usually the case in climate research, especially in paleoclimate studies (Emile-Geay, 2016; Mudelsee, 2014; Weedon, 2003). The most common way of tackling this problem is to interpolate the original unevenly spaced climate time series in the time domain so as to obtain equidistance and the same times. The series can then be analysed using existing conventional correlation analysis techniques. However, experience shows that interpolation has its drawbacks: depending on the features of the method applied, the interpolated time series may show deviations in terms of variability or noise properties, and additional serial dependence may be introduced (Horowitz, 1974; Mudelsee, 2014; Olafsdottir and Mudelsee, 2014). Thus, interpolation should be avoided as far as possible.

Fortunately, there are some algorithms and software available to carry out this task, at least for unevenly spaced climate time series sampled at identical points in time (Mudelsee, 2003; Olafsdottir and Mudelsee, 2014). However, there are few statistical techniques for estimating the correlation between two time series not sampled at identical points in time and their corresponding computational implementations. One exception is the Gaussian-Kernel-based cross-correlation (gXCF) method and its associated software named NESTOOLBOX (Rehfeld et al., 2011; Rehfeld and Kurths, 2014; Rehfeld and Bedartha, 2014) and the extended version (Roberts et al., 2017) that includes a confidence interval obtained by a bootstrapping resampling approach; another exception is binned correlation as proposed by Mudelsee $(2010,2014)$. However, the software for this method is not freely available on the Internet.

Binned correlation is a statistical technique developed to estimate the correlation between two unevenly spaced time series sampled at different points in time. It is also applicable to two evenly spaced time series that are not on the same time grid (Mudelsee, 2014). It is performed by resampling the time series into time bins on a regular grid, and then assigning the mean values of the variable under scrutiny within those bins. Mudelsee (2010) proposes a novel approach adapting the binned correlation technique (used mainly with astronomical data) to analyse climate time series taking into account their memory (or persistence), which is a genuine property of climate time series. Autocorrelation, persistence, memory or serial dependence is characteristic of weather and climate fluctuations, and is recorded in climate time series (Wilks, 2011; Mudelsee, 2002). A simple persistence model used to "represent" climate time series is a first-order autoregressive (AR1) process where a fluctuation depends only on its own immediate past plus a random component (Gilman et al., 1963; Mann and Lees, 1996; Mudelsee, 2002). However, paleoclimate time series are usually unevenly spaced in time, and it is necessary to use an AR1 version for the case of uneven spacing, such as the method proposed by Robinson (1977). The technique of Mudelsee (2010) requires the concept of nonzero persistence times, enabling the mixing information (i.e. covariance) to be recovered, even when the two timescales differ. The BINCOR package presented in this paper is based on a method that is not applicable when one or both of the time series under examination have zero persistence. Similarly, this method is not 
applicable when the time series are sampled with significantly longer spacing than the persistence time, so that the effectively sampled persistence time is zero. A fundamental condition for using this method is that the time spacing should not be much larger than the persistence times. Enough common data points then fall within a time bin, and knowledge can be acquired on the covariance (Mudelsee, 2010, 2014).

In this paper we present a computational package named BINCOR (BINned CORrelation), which is based on the approach proposed by Mudelsee $(2010,2014)$. The BINCOR package contains (i) a main function named bin_cor, which is used to convert the irregular time series to a binned time series; (ii) two complementary functions (cor_ts and ccf_ts) for computing the correlation between the two binned climate time series obtained with the bin_cor function; and (iii) an additional function (plot_ts) for plotting the "primary" vs. the binned time series. This package is programmed in R language and is available at the CRAN repository (https://CRAN. R-project.org/package=BINCOR).

This paper is divided into four sections. The first outlines the method and the computational program. The second presents a Monte Carlo experiment to study the effect of binning size selection. In the Examples section we apply BINCOR to a couple of unevenly spaced real-world climate data sets: instrumental and paleoclimate. Finally, the Summary section presents our main conclusions.

\section{The BINCOR package}

\section{The method}

In this section we outline the main mathematical ideas behind the binned correlation technique for unevenly spaced sampled at different points in time, following the methodology introduced by Mudelsee $(2010,2014)$. The procedure is described as follows:

1. Input: two unevenly spaced climate time series $\left\{X(i), T_{X}\right\}_{i=1}^{N_{X}}$ and $\left\{Y(i), T_{Y}\right\}_{i=1}^{N_{Y}}$, where $T_{X}, T_{Y}$ and $N_{Y}, N_{Y}$ are the time domains and the sample sizes of each series, respectively.

2. Compute the average spacing between samples

- $\bar{d}_{X}=\left[T_{X}\left(N_{X}\right)-T_{X}(1)\right] /\left(N_{X}-1\right)$

- $\bar{d}_{Y}=\left[T_{Y}\left(N_{X}\right)-T_{Y}(1)\right] /\left(N_{Y}-1\right)$

- $\bar{d}_{X Y}=\left[\bar{T}_{\max }-\bar{T}_{\min }\right] /\left(N_{X}+N_{Y}-1\right)$

where $\bar{T}_{\max }=\max \left[T_{X}\left(N_{X}\right), T_{Y}\left(N_{Y}\right)\right]$ and $\bar{T}_{\min }=\min \left[T_{X}(1), T_{Y}(1)\right]$.

3. Estimate the bin-width $(\bar{\tau})$ taking into account the persistence (memory) estimated for each unevenly spaced climate time series, $X$ and $Y$ denoted as $\hat{\tau}_{X}$ and $\hat{\tau}_{Y}$, respectively. To estimate the persistence, an AR1 model (Robinson, 1977) is fitted to each unevenly spaced time series (Mudelsee, 2002). BINCOR includes three rules for estimating the bin-width (the options are shown in Table 1), but we prefer to use rule number 3 as the default value (FLAGTAU=3) because in terms of the RMSE (Section Monte Carlo experiments) of this rule Monte Carlo simulations are superior to the other rules for estimating the bin-width (Mudelsee, 2014).

- Estimate the bias-corrected equivalent autocorrelation coefficients

$$
\hat{\bar{a}}_{X}^{\prime}=\exp \left(-\bar{d}_{X} / \hat{\tau}_{X}^{\prime}\right), \hat{\bar{a}}_{Y}^{\prime}=\exp \left(-\bar{d}_{Y} / \hat{\tau}_{Y}^{\prime}\right), \text { and } \hat{\bar{a}}_{X Y}^{\prime}=\sqrt{\hat{\bar{a}}_{X}^{\prime} \cdot \hat{\bar{a}}_{Y}^{\prime}}
$$

- Estimate the bin-width as $\bar{\tau}=-\bar{d}_{X Y} / \ln \left(\hat{\bar{a}}_{X Y}^{\prime}\right)$ (Eq. 7.48 in Mudelsee (2002)), the default option (FLAGTAU=3) in the BINCOR package, other options are:

\begin{tabular}{rcr}
$\bar{\tau}$ rule & FLAGTAU option & Reference \\
\hline$\tau_{x}+\tau_{y}$ & 1 & Eq. 7.44 in Mudelsee (2014) \\
$\max \left(\tau_{x}, \tau_{y}\right)$ & 2 & Eq. 7.45 in Mudelsee (2014) \\
$-\bar{d}_{X Y} / \ln \left(\hat{\bar{a}}_{X Y}^{\prime}\right)$ & 3 & Eq. 7.48 in Mudelsee (2014) \\
\hline
\end{tabular}

Table 1: The FLAGTAU options and its corresponding methods (rules) to estimate the bin-width.

4. Determine the number of bins: $N_{b}=\left(\bar{T}_{\max }-\bar{T}_{\min }\right) / \bar{\tau}$

5. Set: $\lim _{\text {inf }}(n=1)=\bar{T}_{\text {min }}$. Then, for $n=1,2, \ldots, N_{b}$, define (Figure 1$)$ :

(a) $\lim _{\sup }(n)=\bar{T}_{\min }+n \cdot \bar{\tau}$ 
(b) $\operatorname{id} T_{X}=\mathrm{WHICH}\left[T_{X} \geq \lim _{\text {inf }}(n) \operatorname{AND} T_{X} \leq \lim _{\text {sup }}(n)\right]$

(c) $\operatorname{id} T_{Y}=\mathrm{WHICH}\left[T_{Y} \geq \lim _{\text {inf }}(n)\right.$ AND $\left.T_{Y} \leq \lim _{\text {sup }}(n)\right]$

(d) $\mathrm{LT}_{X}=\operatorname{LENGTH}\left(\mathrm{id} T_{X}\right)$

(e) $\mathrm{LT} T_{Y}=\operatorname{LENGTH}\left(\mathrm{id} T_{Y}\right)$ if $\left(\mathrm{LT}_{X}>0 \mathrm{AND} \mathrm{L} T_{Y}>0\right)$

i. $F(n)=$ mean of $X\left(\mathrm{id} T_{X}\right)$

ii. $G(n)=$ mean of $Y\left(\operatorname{id} T_{Y}\right)$

iii. $T(n)=\left[\lim _{\text {inf }}(n)+\lim _{\text {sup }}(n)\right] / 2$

(f) $\lim _{\text {inf }}(n)=\lim _{\text {sup }}(n)$

6. Output: two binned climate time series $\left\{T_{n}, F(n)\right\}_{n=1}^{N_{b}}$ and $\left\{T_{n}, G(n)\right\}_{n=1}^{N_{b}}$, where $N_{b}$ is the number of bins.

7. Estimate the correlation between the two binned time series. This can be done through the native $\mathrm{R}$ functions cor and ccf or by means of the BINCOR functions cor_ts and ccf_ts.

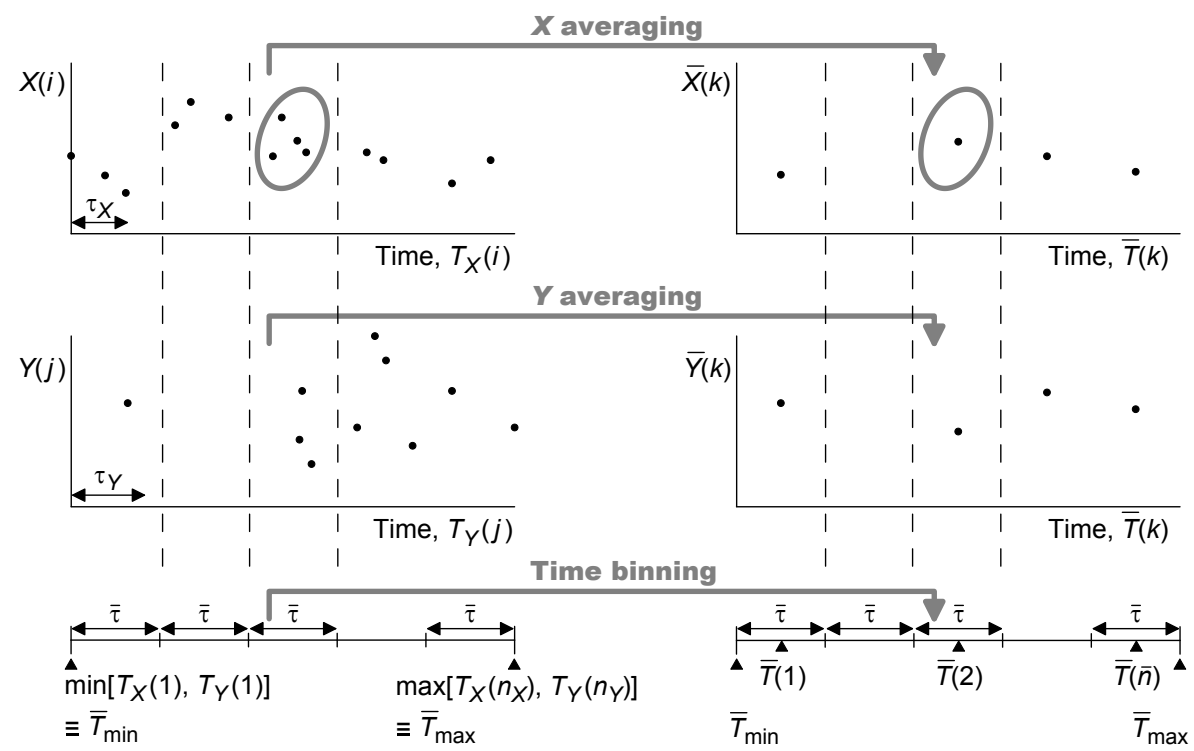

Figure 1: Graphical representation for the binned correlation procedure presented in Step 5. Modified from (Mudelsee, 2010, 2014).

\section{Monte Carlo experiments}

We conducted Monte Carlo experiments to study how the specific rules (Table 1) chosen for calculating the bin-width based on persistence reduce the error compared to arbitrarily choosing a bin-width. The parameter configuration for the Monte Carlo experiments is presented in Figure 2. To carry out the Monte Carlo simulations, we used the bivariate Gaussian AR1 process for uneven time spacings (Mudelsee, 2014), which is given by

$$
\begin{array}{r}
X(1)=\mu_{N(0,1)}^{X}(1), \\
Y(1)=\mu_{N(0,1)}^{Y}(1), \\
X(t)=a_{X} X(t-1)+\mu_{N\left(0,1-a_{X}^{2}\right)}^{X}(t), \quad t=2, \ldots, N, \\
Y(t)=a_{Y} Y(t-1)+\mu_{N\left(0,1-a_{Y}^{2}\right)}^{Y}(t), \quad t=2, \ldots, N,
\end{array}
$$

where $a_{X}$ and $a_{Y}$, the autoregressive parameters for $X(t)$ and $Y(t)$, are defined as (Mudelsee, 2014): $a_{X}=\exp \left\{-\left[T_{X}(t)-T_{X}(t-1)\right] / \tau_{X}\right\}$ and $a_{Y}=\exp \left\{-\left[T_{Y}(t)-T_{Y}(t-1)\right] / \tau_{Y}\right\}$. The correlation (by construction) between $X(t)$ and $Y(t)$ is $\rho_{X Y}$ (see Mudelsee, 2014, pp. 307-309 for more details about the statistical properties of the bivariate AR1 process for unevenly spaced time series). To generate the uneven timescales for $X(i)$ and $Y(j)$, we follow the methodology proposed by (see Mudelsee, 2014, 
pp. 299-304), which consists of producing a number $(10 \mathrm{~N})$ of data pairs on an evenly spaced grid of 1.0 , discarding $90 \%$ of points and retaining $10 \%$ of $X$ and $Y\left(N_{x}=N_{y}=N\right)$ points. The time points for $X(i)$ and $Y(j)$ are subject to the following conditions:

1. Control case (equal timescales):

- Condition 1: $N_{X}=N_{Y}$

- Condition 2: $\left\{T_{X}(i)\right\}_{i=1}^{N_{X}}=\left\{T_{Y}(j)\right\}_{j=1}^{N_{Y}}$

2. "Well" mixed unequal timescales:

- Condition 1: $T_{X}(i) \neq T_{Y}(j)$ for all $i$ and $j$

- Condition 2: $T_{X}(1)<T_{Y}(1)<T_{X}(2)<T_{Y}(2)<T_{X}(3)<\ldots<T_{X}\left(N_{X}\right)<T_{Y}\left(N_{Y}\right)$

3. "Wildly" mixed unequal timescales:

- There are not conditions for this case.
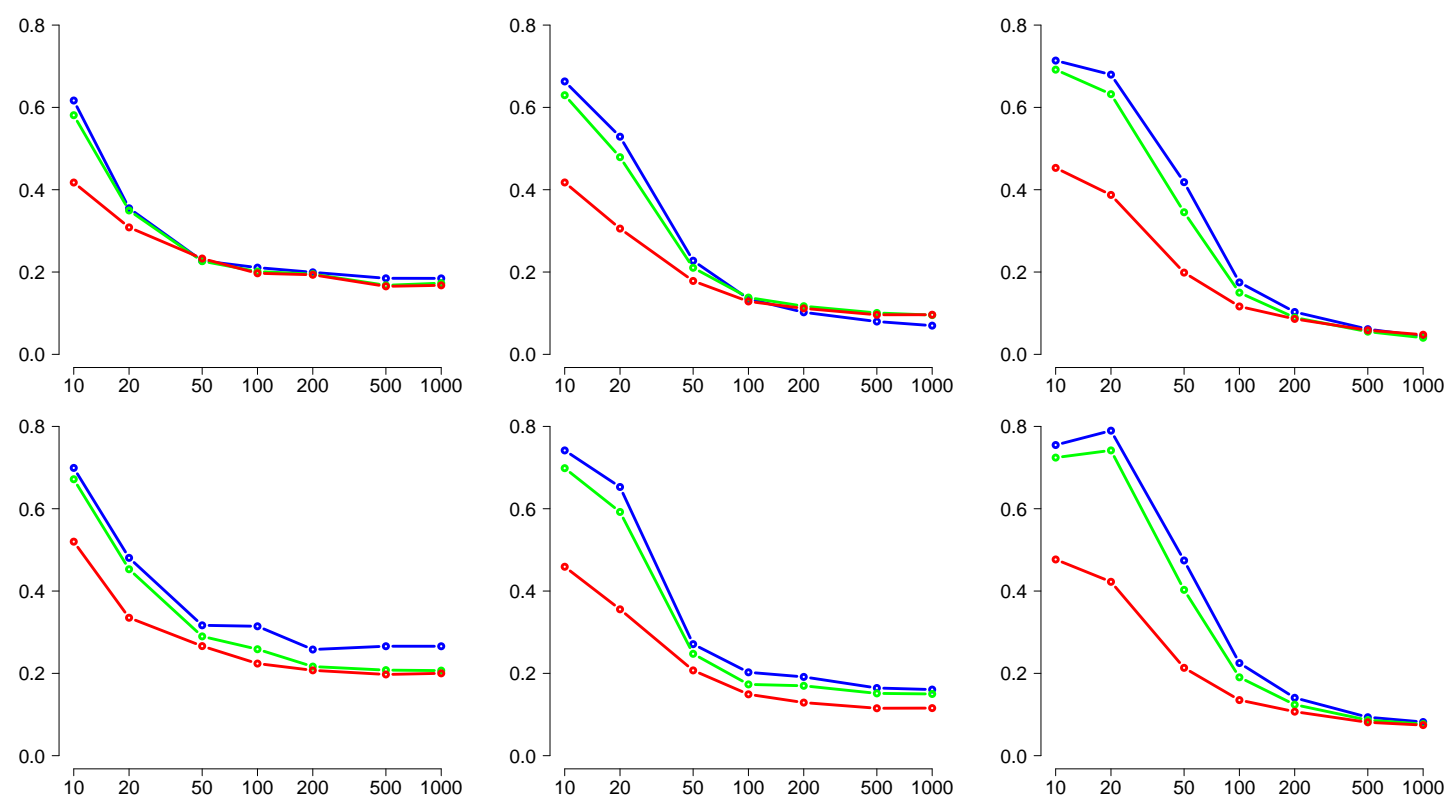

Figure 2: Monte Carlo experiments to test the impact of the rules (Table 1) used to calculate the bin-width and their role in the estimation of the binned correlation. The persistence figures for $X$ and $Y$ are 10 (column 1), 20 (column 2) and 50 (column 3), respectively. The constraints for the resampling timescales are for well mixed (first row) and wildly mixed (second row) cases. The horizontal axis indicates the sample sizes (in $\log 10$ scale) and the vertical axis shows the RMSE that is determined via averaging $\left(\hat{\rho}_{X Y}-\rho_{X Y}\right)^{2}$ over 5,000 simulations. The blue, green and red curves indicate rules 1 (sum), 2 (max) and 3 (the default rule option in BINCOR).

The outcome of the Monte Carlo experiments is as follows: 1) For equal timescales (figures not shown), all three rules behave similarly (as expected) in terms of RMSE, although the RMSE increases slightly as the persistence increases. 2) The well mixed case shows that for RMSE the rules take two different "patterns" with the first two rules (sum and max) on one hand and the third rule (the default rule option) on the other. This difference is most noticeable in the first values of the samples (from 10 to 100) and is most pronounced with high persistence values $\left(\tau_{x}\right.$ and $\left.\tau_{y}\right)$. The rule that shows the smallest RMSE is rule 3 (the default option), though it is important to point out that for $\tau_{x}=\tau_{y}=50$ the RMSE figures are practically indistinguishable for sample sizes from 200 to 1000. 3) Finally, RMSE in the wildly mixed case behaves more or less similarly to the well mixed case, though rule 3 yields the smallest RMSE for all three persistence values. Bearing in mind that the wildly mixed case does not impose conditions on generating timescales, and in practice the unevenly spaced climate time series could contain some degree of randomness in the sampling times, the best rule in terms of RMSE for estimating bin-width $(\bar{\tau})$ and binned correlation can be said to be number 3, i.e. the default rule used in BINCOR to estimate the bin-width. 


\section{The computer program}

The BINCOR package developed in R version 3.1.2 ${ }^{1}$ to be run from the command line runs on all major operating systems and is available from the CRAN repository (http: //CRAN. R-project.org/ package=BINCOR). The BINCOR package contains four functions: 1) bin_cor (the main function for building the binned time series); 2) plot_ts (for plotting and comparing the "primary" and binned time series); 3) cor_ts (for estimating the correlation between the binned time series); and 4) ccf_ts (for estimating the cross-correlation between the binned time series). The graphical outputs can be displayed on the screen or saved as PNG, JPG, or PDF graphics files. BINCOR depends on the dplR (Bunn et al., 2015) and pracma (Borchers, 2015) packages. The dplR package is used by the function bin_cor to calculate the persistence for the climate time series under study, whereas the pracma package is used by the functions cor_ts and ccf_ts to remove the linear trend before estimating the correlation.

The first (and main) function, bin_cor, estimates the binned time series taking into account the memory or persistence of the unevenly spaced climate time series to be analysed (Mudelsee, 2002). It has the following syntax:

R> bin_cor(ts1, ts2, FLAGTAU=3, ofilename),

where

- ts1 and ts2 are unevenly spaced time series.

- FLAGTAU defines the method used to estimate the bin-width $(\bar{\tau})$. There are three methods included in BINCOR for estimating bin-width (Table 1 ), but we prefer to use (FLAGTAU $=3$ ) as the default rule because Monte Carlo simulations perform better in terms of RMSE than the other rules in estimating the bin-width and the binned correlations (Mudelsee, 2014).

- 'ofilename' is the name of the output file (in ASCII format) which contains the binned time series.

bin_cor returns a list object containing the following outputs:

"Binned_time_series", "Auto._cor._coef._ts1", "Persistence_ts1", "Auto._cor._coef._ts2", "Persistence_ts2", "bin width", "Number_of_bins", "Average spacing", "VAR. ts1", "VAR. bin ts1", "VAR. ts2", "VAR. bin ts2", "VAR. ts1 - VAR bints1",

"VAR. ts2 - VAR bints2", "\% of VAR. lost ts1", "\% of VAR. lost ts2".

The names of the outputs are self-explanatory, but we wish to highlight that Average spacing is the mean value of the times for the binned time series; VAR. ts1, VAR. bin ts1, VAR. ts2 and VAR. bin ts 2 are the variances for ts 1 and ts 2 for their respective binned time series; the next two outputs are the differences between the variances of $\mathrm{ts} 1$ and $\mathrm{ts} 2$ and their corresponding binned time series; and the last two outputs are the percentages of variance lost for $t s 1$ and $t s 2$ as a result of the binned process.

The second function, called plot_ts, plots the "primary" (unevenly spaced) time series and the binned time series. The plot_ts function contains the following elements:

R> plot_ts(ts1, ts2, bints1, bints2, varnamets1="", varnamets2="", colts $1=1, \operatorname{colts} 2=1$, colbints $1=2$, colbints $2=2$, ltyts $1=1$,

ltyts $2=1$, ltybints1=2, 1tybints2=2, device="screen", ofilename),

where the input arguments ts 1 and ts 2 are the unevenly spaced time series, bints 1 and bints 2 are the binned time series, varnamets 1 and varnamets 2 are the names of the variables under study, colts 1 , colts2 (by default both curves are in black) and colbints1, colbints2 (by default both curves are in red) are the colours for the "primary" and binned times series; 1tyts1, 1tyts2, 1tybints 1 and 1 tybints 2 are the types of line to be plotted for the "primary" and binned times series, respectively ( 1 = solid, 2 = dashed, 3 = dotted, $4=$ dot-dashed, 5 = long-dashed, $6=$ double-dashed); device is the type of output device ("screen" by default, the other options being "jpg," "png," and "pdf"); resfig is the image resolution in "ppi" (by default $\mathrm{R}$ does not record a resolution in the image file, except for $\mathrm{BMP} ; 150$ ppi could be a suitable value); 'ofilename' is the output filename; and finally, $\mathrm{Hfig}$, WFig and Hpdf, Wpdf are the height and width of the output for the JPG/PNG and PDF formats, respectively.

The third function, cor_ts, calculates three types of correlation coefficient: Pearson's correlation, Spearman's and Kendall's rank correlations. These correlation coefficients are estimated through the native R function cor. test from the R package Stats. The cor_ts function has an option to remove the linear trend of the time series under analysis - other pre-processing methods could be used before the cor_ts function is applied. This function has the following syntax:

\footnotetext{
${ }^{1}$ It was also tested in R 3.4.1.
} 
R> cor_ts(bints1, bints2, varnamets1="", varnamets2="",

KoCM, rmltrd="N", device="screen", Hfig, Wfig, Hpdf, Wpdf,

resfig, ofilename)

where KoCM indicates the correlation estimator: pearson for Pearson (the option by default), spearman for Spearman and kendall for Kendall; rmltrd is the option to remove the linear trend in the time series under study (by default the linear trend is not removed, but the function can be enabled via the option " $Y$ " or " $y$ "). The other parameters are described some lines above. cor_ts has as its output a list object containing the main information for the estimated correlation coefficient (e.g. a 95\% confidence interval for Pearson and a p-value for Spearman and Kendall). The cor_ts function also provides a scatterplot for the binned time series, which can be plotted on the screen (by default) or saved in JPG, PNG or PDF formats (the parameter 'ofilename' is available to assign a name to this output).

Finally, the fourth function, ccf_ts, estimates and plots the cross-correlation between two evenly spaced paleoclimate time series. We use the native R function ccf (R Stats package) to estimate the cross-correlation in our ccf_ts function. The ccf_ts function has the following syntax:

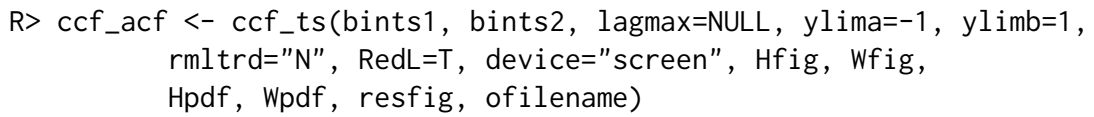

All these elements are already defined above except the parameters lagmax $=N U L L, y l i m a=-1, y l i m b=1$ and RedL. The first parameter indicates the maximum lag for which the cross-correlation is calculated (its value depends on the length of the data set), the next two parameters indicate the extremes of the range in which the CCF will be plotted and the last parameter (the default option is TRUE) plots a straight red line to highlight the correlation coefficient at lag 0 . The ccf_ts function generates as its output the acf (auto-correlation function; ACF) R object, which is a list with the following parameters: lag is a three dimensional array containing the lags at which the ACF is estimated; acf is an array with the same dimensions as lag containing the estimated ACF; type is the type of correlation (correlation (the default), covariance and partial); $n$. used is the number of observations in the time series; and snames provides the names of the time series (bints1 and bints2).

\section{Examples}

\section{Assessing the link between El Niño-Southern Oscillation and Northern Hemisphere sea surface temperature}

We first examine two evenly-spaced annually-resolved instrumental climate records that cover the time interval from 1850 to $2006(N=157 \text { points })^{2}$. To test our BINCOR package we created irregular time series by randomly removing $20 \%$ of the data from the evenly spaced time series. We note that the new "sampling" times are not necessarily the same for both irregular series. The new irregular time series ("primary" hereafter) consist of 125 data points and have an average temporal spacing $\bar{d}$ of 1.24 years. Specifically the two time series used were a record of Northern Hemisphere (NH) sea surface temperature (SST) anomalies (HadCRUT3, Brohan et al. (2006)) and a record of equatorial Pacific SST anomalies from the El Niño 3 region $\left(2.5^{\circ} \mathrm{S}\right.$ to $2.5^{\circ} \mathrm{N}, 92.5$ to $147.5^{\circ} \mathrm{W}$ ) (Mann et al., 2009), which is a indicator of El Niño-Southern Oscillation (ENSO). Both time series, especially the NH-SST data, show strong autocorrelation (plots not shown) and long-term trends (inspected by Mann-Kendall test; ENSO, $z=6.52$ and $p$-value $<0.001$ and NH-SST, $z=10.214$ and $p$-value $<0.001$ ). To generate the sample data, we fit a linear model to each evenly spaced time series and, after removing the model fitted to the evenly spaced data, we use the residuals (i.e. the difference between the observed data and the model fitted) to build the irregular time series and then create the binned time series.

The code used to generate Figure 3 is shown below.

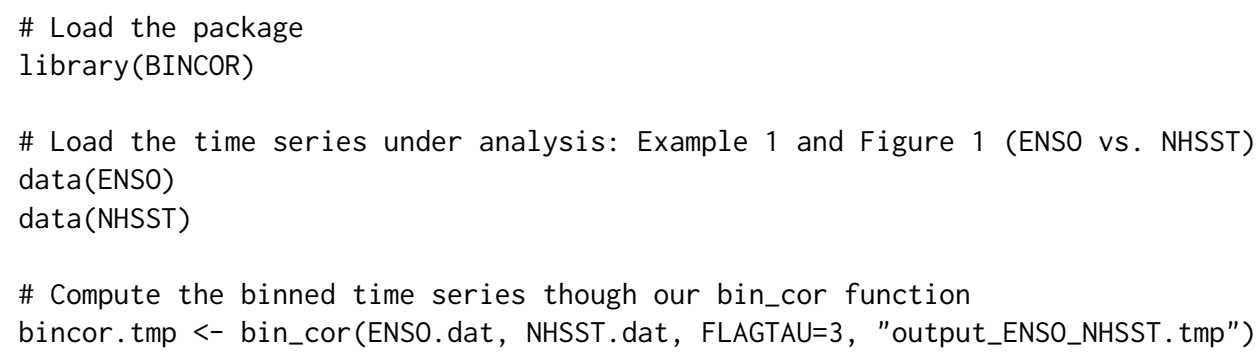

\footnotetext{
${ }^{2}$ The data sets can be obtained from the following URL http://www. meteo.psu. edu/holocene/public_html/ supplements/MultiproxySpatialog/results/ (NINO3 full and Northern Hemisphere full).
} 
A)

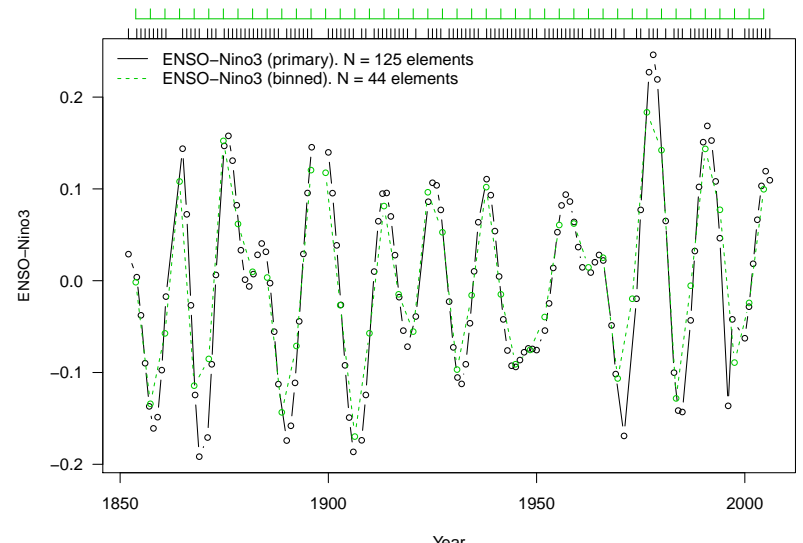

B)

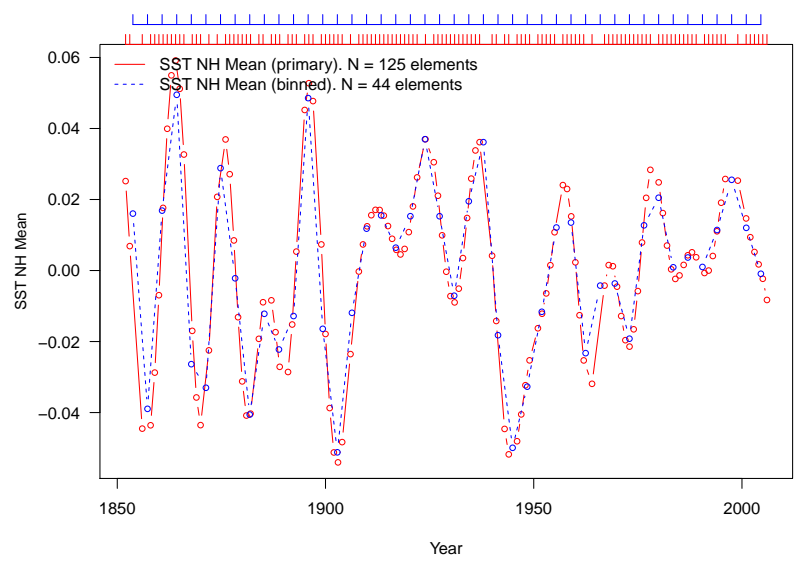

C)

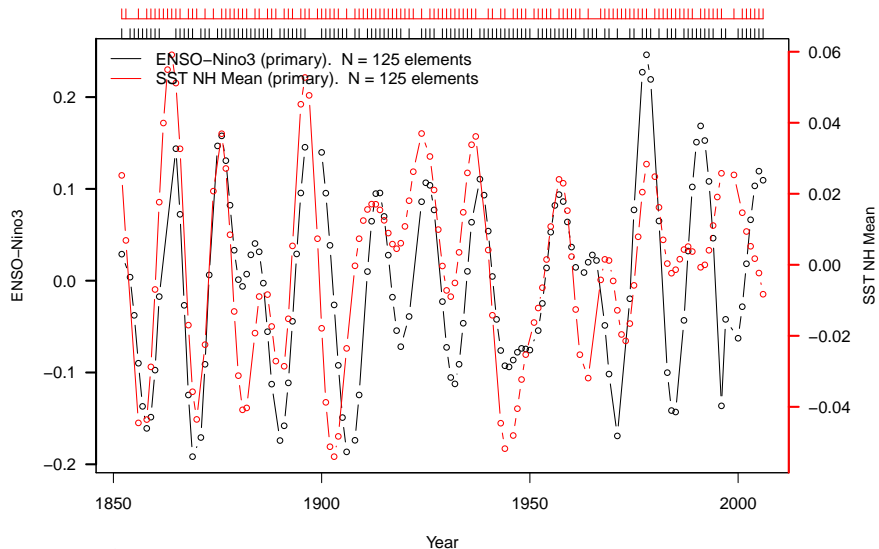

D)

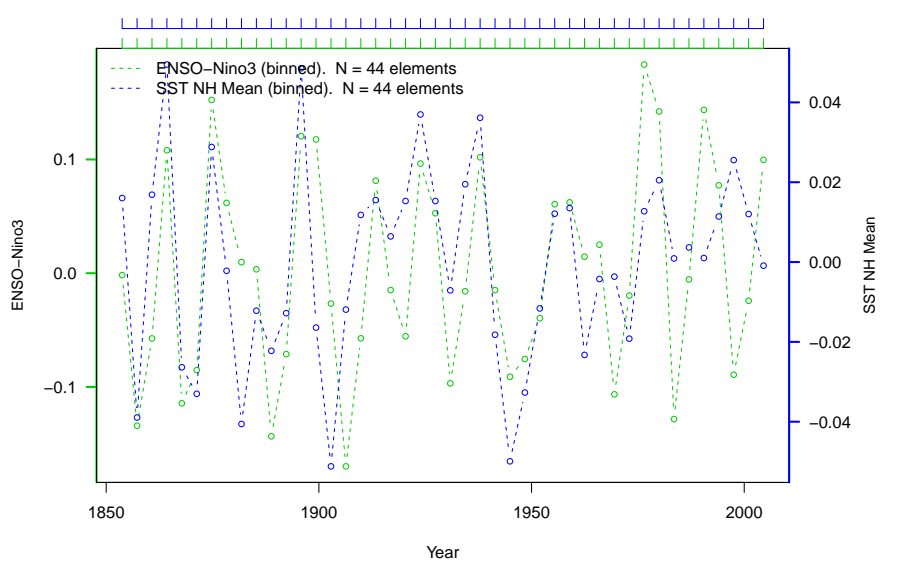

Figure 3: "Primary" (unevenly spaced) and binned ENSO-Niño3 (Mann et al., 2009) and NH-SST (Brohan et al., 2006). The autocorrelation and persistence values for ENSO are $\hat{a}^{\prime}=0.82$ and $\hat{\tau}=6.25$ years, and for NH-SST are $\hat{a}^{\prime}=0.86$ and $\bar{\tau}=8.05$ years. The horizontal top axes indicate the sampling times for the plotted time series.

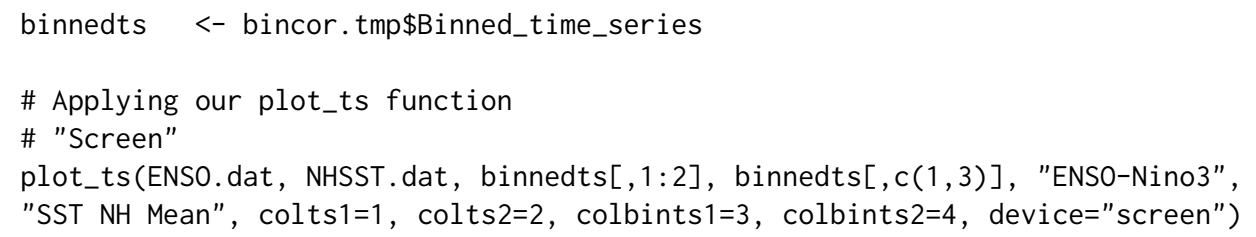

Figures $3 \mathrm{~A}$ and $3 \mathrm{~B}$ show the binned time series (ENSO in green and NH-SST in red) obtained with our bin_cor function. Although we use residuals, they show a relative high autocorrelation $\left(\hat{a}_{\text {ENSO }}^{\prime}=0.82\right.$ and $\left.\hat{a}_{\mathrm{SST}}^{\prime}=0.86\right)$ and their corresponding estimated bias-corrected persistence values are $\hat{\tau}_{\text {ENSO }}=6.25$ years and $\bar{\tau}_{\mathrm{SST}}=8.05$ years. The number of bins and, thus, the number of elements for each binned time series is 44 and the distance between elements is 3.5 years. We also plot the "primary" climate time series (in black) to compare them with the binned series. Visually, the binned time series are roughly similar to the "primary" series. This observation is also supported by the statistical similarity method (Frentzos et al., 2007) as implemented in the R package TSdist (Mori et al., 2015, 2016). The dissimilarity metric (DISSIM) has the following interpretation: a value of zero indicates a perfect relationship such that the closer DISSIM is to zero, the more similar are the time series. The DISSIM between the binned and "primary" ENSO time series and the binned and "primary" NH-SST series are 3.70 and 0.84 , respectively. This corroborates the similarity between the "primary" and binned time series observed visually. Figure 3 also shows a comparison between the "primary" climate time series (Figure 3 C) and the binned series (Figure 3 D). Note that this plot shows that the number of elements $(N=125)$ is the same for both "primary" series, but this is not strictly necessary: our bin_cor function is able to tackle time series with different numbers of elements.

The second result obtained from our BINCOR package, and more specifically from the cor_ts 
function, is shown in Figure 4, which shows the scatterplot between the ENSO (x-axis) and NH-SST (y-axis) binned time series. This scatterplot shows a moderate increasing trend from left to right, suggesting a potentially positive relationship between the two binned time series. This pattern can be confirmed statistically by means of the cor_ts function output, which also provides the correlation coefficient between two time series under analysis. For this case, the Pearson's correlation (with $95 \%$ confidence interval) obtained is $\bar{r}_{X Y}=0.53[0.28 ; 0.71]$ (other estimators can also be used in cor_ts). This value is close to the Pearson's correlation estimated for the evenly spaced climate time series, which is $\bar{r}_{X Y}=0.58$ [0.46; 0.67]. The relatively high correlation obtained between these two climate records is expected; ENSO-related climate variability is observed in many regions outside the equatorial Pacific, particularly in the tropical North Atlantic (Enfield and Mayer, 1997; Garcia-Serrano et al., 2017).

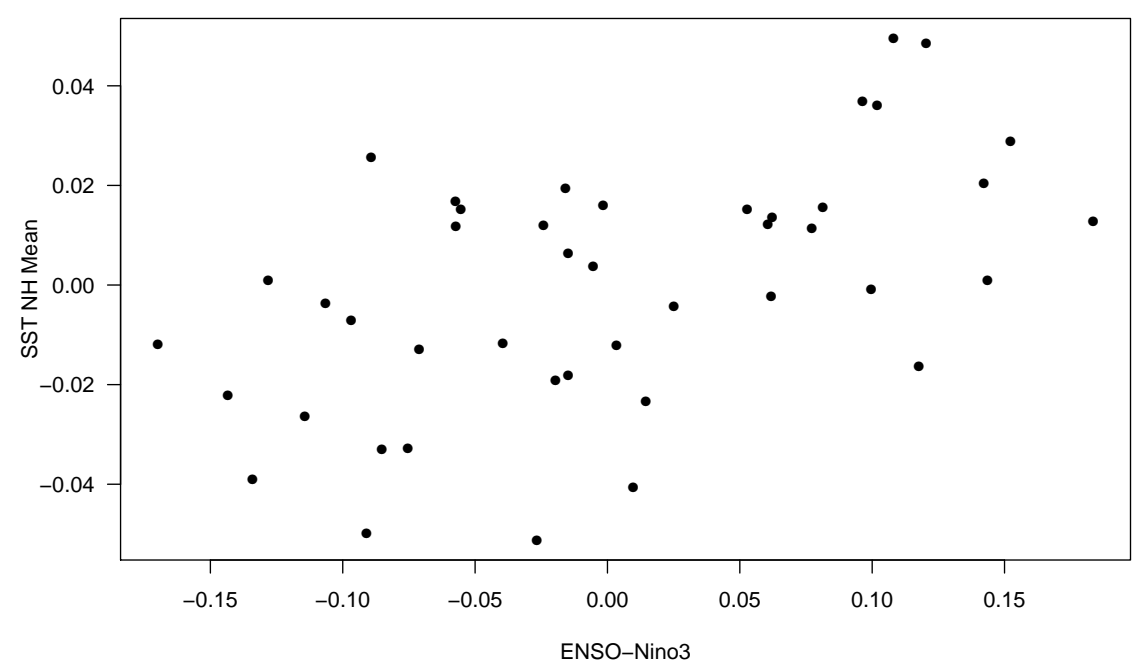

Figure 4: Scatterplot for the ENSO-Niño3 (Mann et al., 2009) and NH-SST (Brohan et al., 2006) binned time series. The Pearson's correlation coefficient (with 95\% confidence interval) is $\bar{r}_{X Y}=0.53[0.28$; 0.71].

The code used to generate Figure 2 is shown below.

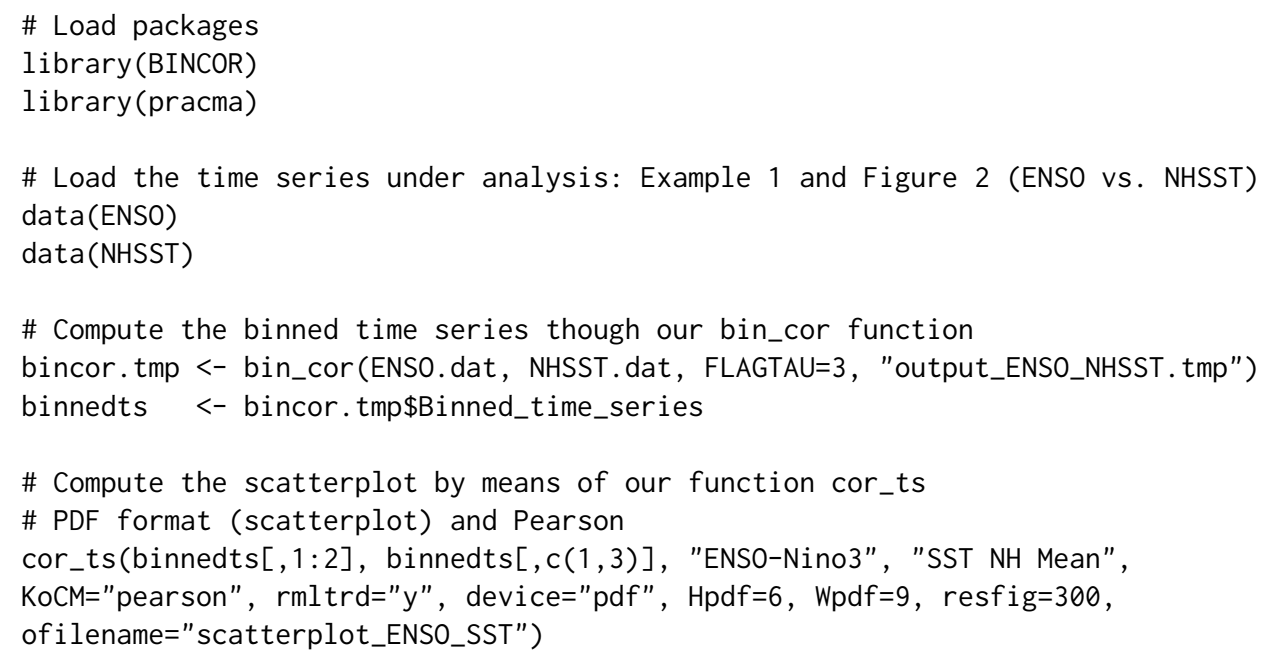

\section{Abrupt climate changes during the last glacial}

We report an analysis of two temporally unevenly-spaced pollen records from two marine sediment cores (MD04-2845 and MD95-2039) ${ }^{3}$ collected on the south-western European margin (Figure 5).

\footnotetext{
${ }^{3}$ The data sets can be obtained from https://doi.pangaea.de/10.1594/PANGAEA.870867. These time series come from a global pollen and charcoal database (?) drawn up under the framework of the INQUA International
} 
The aim of this case study is to show the use of BINCOR to estimate the correlation between two unevenly spaced paleoclimate time series by means of the cross-correlation function. The pollen time series analysed in this example span the interval between 73,000 and 15,000 years before present (BP), thus covering the last glacial period (LGP). The climate during the LGP was characterised by millennial variability with "abrupt" transitions between cold stadials and warm interstadials known as Dansgaard-Oeschger (D-O) cycles (Dansgaard et al., 1993; Wolff et al., 2012). The D-O cycles are characterised by rather fast atmospheric warming events over Greenland of up to $16{ }^{\circ} \mathrm{C}$ that occur within a period of approximately 40 years, followed by gradual cooling leading to the cold stadials (?Wolff et al., 2012).

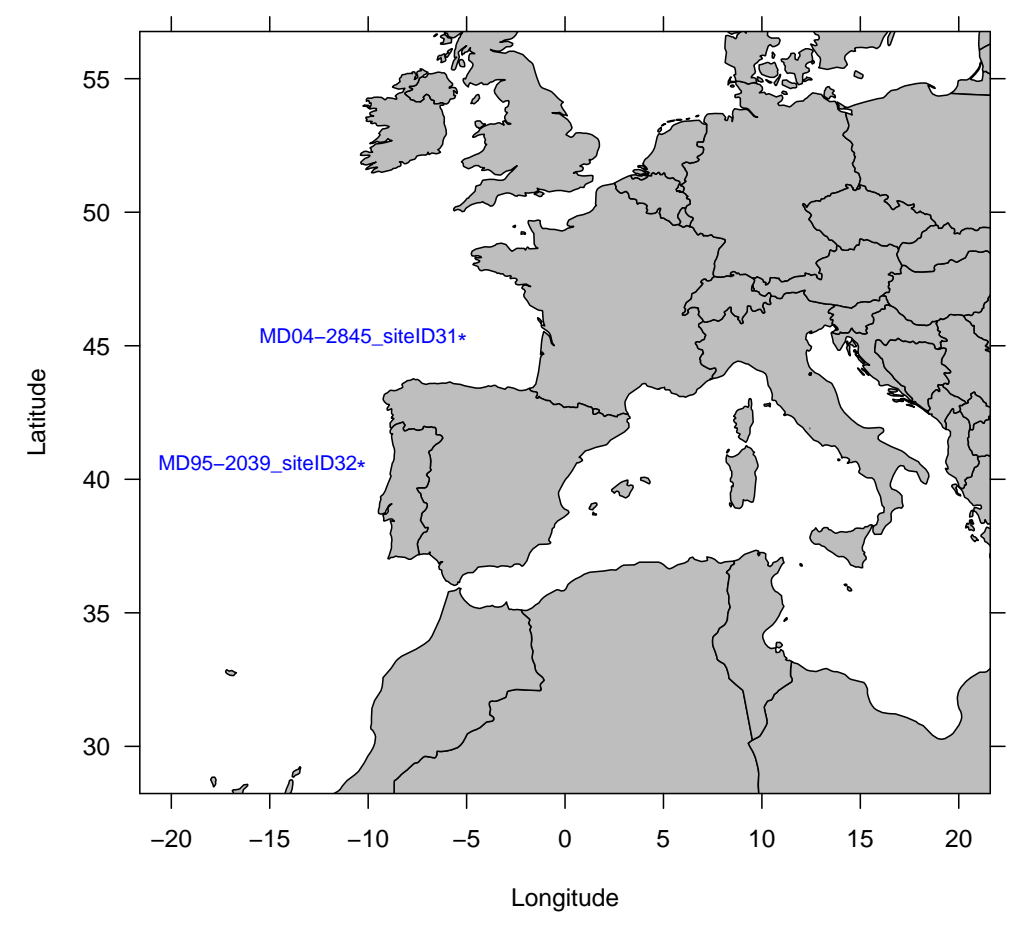

Figure 5: Geographical locations for the pollen time series under analysis (?). The labels indicate the names of the sites where the pollen data were obtained.

Figure 6 illustrates the variations in the pollen percentages of the temperate forest, a type of vegetation typical of moderate, warm, wet climates. Figure 6 A shows the primary and binned pollen records from site MD04-2845 (Sanchez Goni et al., 2008; ?). Figure 6 B shows the primary and binned pollen records from site MD95-2039 (Roucoux et al., 2005; ?). We use the pollen time series with a harmonised, consistent chronology (?) to carry out a fair comparison. We apply our bin_cor and plot_ts functions and obtain the binned time series, which have 27 elements, and a temporal distance between elements of 1220 years. The binned time series show a relatively high level of autocorrelation, $\hat{\bar{a}}_{\mathrm{MD} 04-2845}^{\prime}=0.85$ and $\hat{\bar{a}}_{\mathrm{MD} 95-2039}^{\prime}=0.80$, and an estimated bias-corrected persistence values of $\hat{\tau}_{\text {MD04-2845 }}=3400$ years and $\bar{\tau}_{\text {MD95-2039 }}=1300$ years. It can be observed from Figures 6 A and $6 \mathrm{~B}$ that the binned time series are roughly similar to the "primary" time series, although binning causes some information loss. This is due to the high degree of irregularity in the sampling of the "primary" time series, which makes it difficult to resample when the binned time series are built. In addition, information is lost because the length of the bin is dependent on the persistence and autocorrelation of the "primary" time series. Finally, Figures $6 \mathrm{C}$ and $6 \mathrm{D}$ show that the two pollen time series, presented as the primary and binned data, may be significantly correlated. This is discussed below.

The code used to generate Figure 6 is as follows.

\author{
\# Load the package \\ library (BINCOR) \\ library (pracma) \\ \# Load the time series under analysis: Example 2 and Figure 6
}

Focus Group ACER (Abrupt Climate Changes and Environmental Responses). 
A)

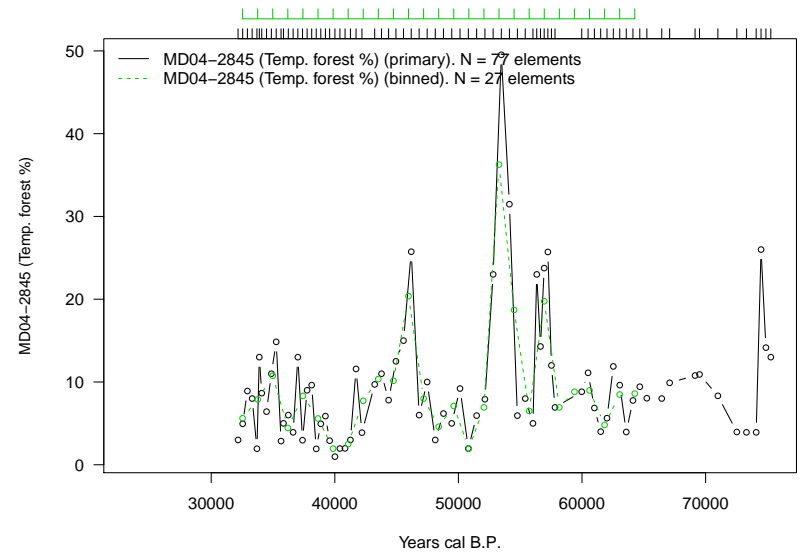

B)

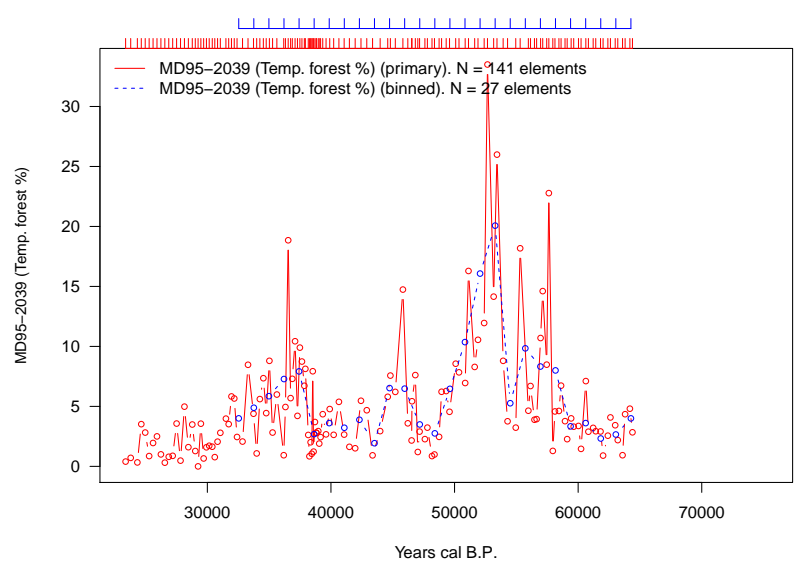

C)

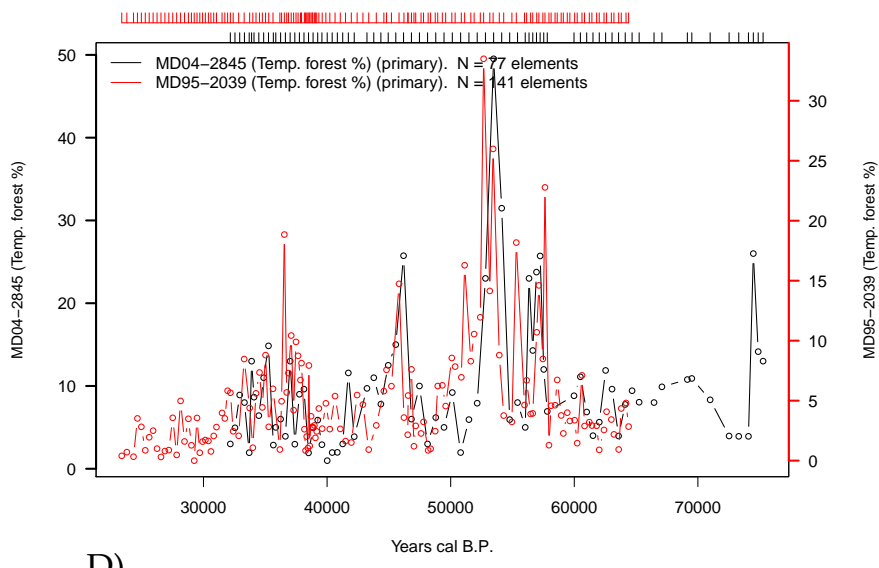

D)

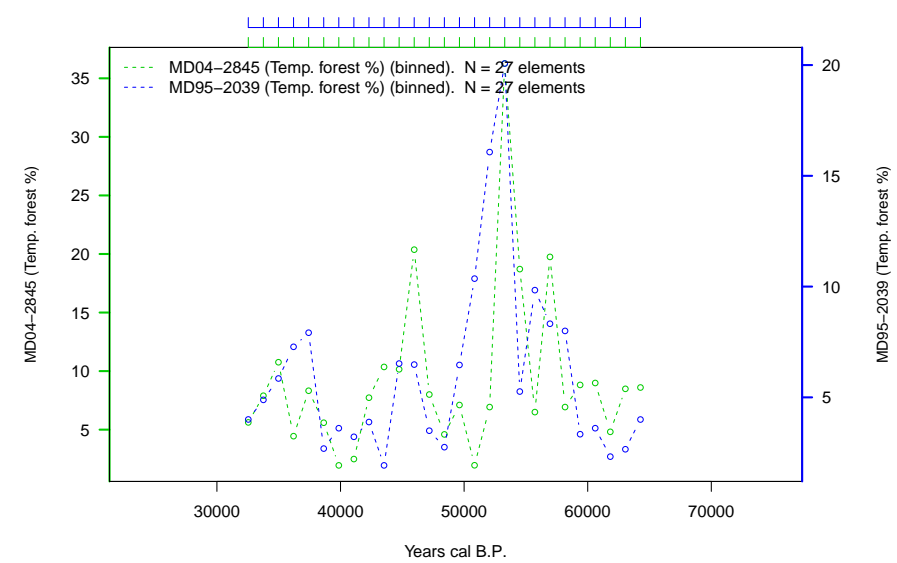

Figure 6: "Primary" (unevenly spaced) and binned pollen time series under analysis (?). The numbers of elements for both time series are provided in the legend. The autocorrelation and persistence values for the time series from site MD04-2845 are $\hat{a}^{\prime}=0.85$ and $\hat{\tau}=3400$ years, and those from site MD95-2039 are $\hat{a}^{\prime}=0.80$ and $\tau=1300$ years. The horizontal top axes indicate the sampling times for the plotted time series.

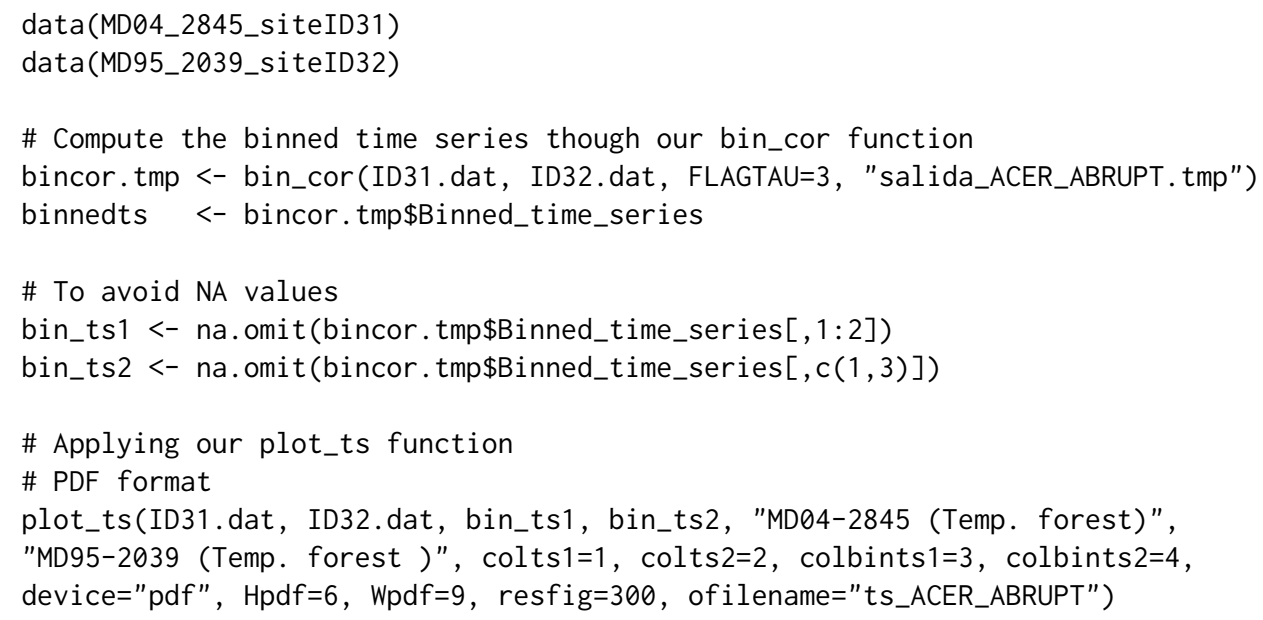

The cross-correlation (CCF) analysis obtained with our ccf_ts function is shown in Figure 7. Before applying the ccf_ts function, a linear trend was removed from the binned time series by enabling the rmltrd option in ccf_ts, and then the residuals were used. The CCF reveals a high correlation $\left(r_{x y}=0.53\right)$ between the binned time series at lag 0 . The high correlation between the pollen records from sites MD04-2845 and MD95-2039 reflects similar responses by vegetation to regional climate variability, particularly to changes in precipitation and temperature. However, the most noticeable result in our CCF analysis is that the maximum correlation $\left(r_{x y}=0.63\right)$ is obtained at lag 1. 
At face value, this result suggests that pollen variability at site MD04-2845 leads that observed at site MD95-2039 by 1220 years. Nevertheless, these sites are located relatively close to each other and are in the same climate domain today, so it is difficult to envisage such a time difference in the response of vegetation (pollen) to rapid climatic changes in the past. The most plausible explanation for this out-of-phase relationship probably lies in the chronological uncertainties of the age models applied to these records. Despite best-efforts to harmonise the different time series in the ACER database using radiometric dating (?), the lack of ${ }^{14} \mathrm{C}$ dates for site MD95-2039 forced us to build the age model for this site by tuning the planktic foraminifera and GRIP ice core oxygen isotopic records (Roucoux et al., 2005). This tuning could affect the time series from site MD95-2039 and introduce unacknowledged chronological uncertainties (Blaauw, 2012; Hu et al., 2017). To summarise, with the present state of data quality we cannot rule out the idea that timescale uncertainties -rather than climate impact adaptation - caused the lag observed.

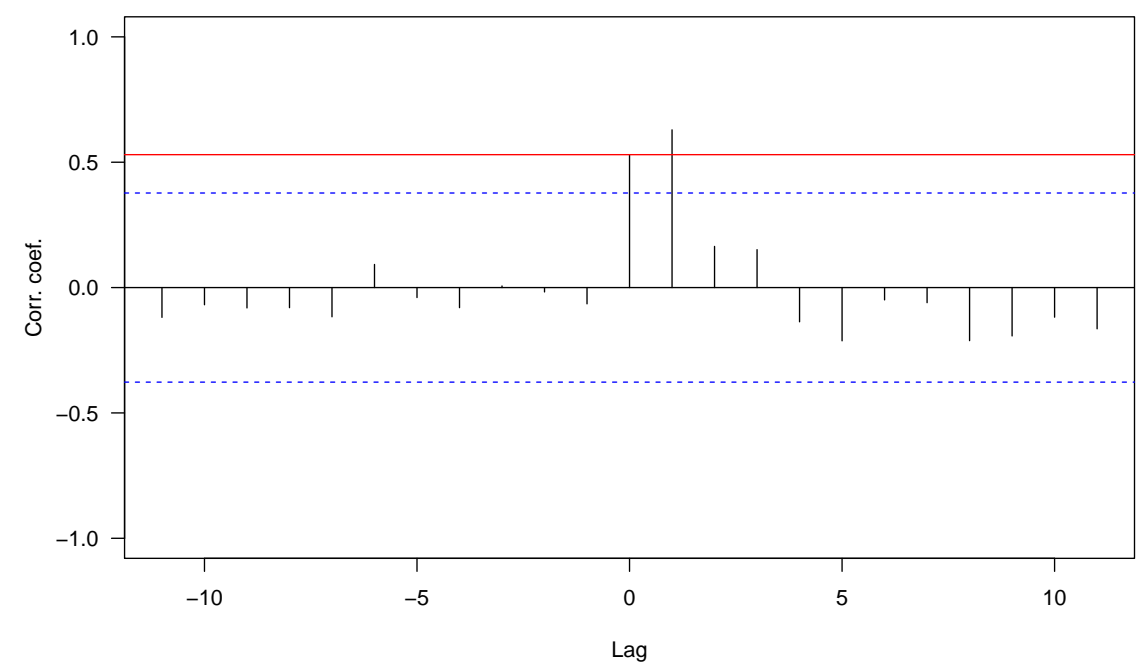

Figure 7: Cross-correlation for the residuals of the binned pollen time series from sites MD04-2845 and MD95-2039 (?). The CCF correlation coefficients at lag 0 and 1 are 0.53 and 0.63 , respectively. The red line indicates the correlation coefficient for lag 0 . Each lag is equivalent to 1220 years.

The code used to generate Figure 7 is the following.

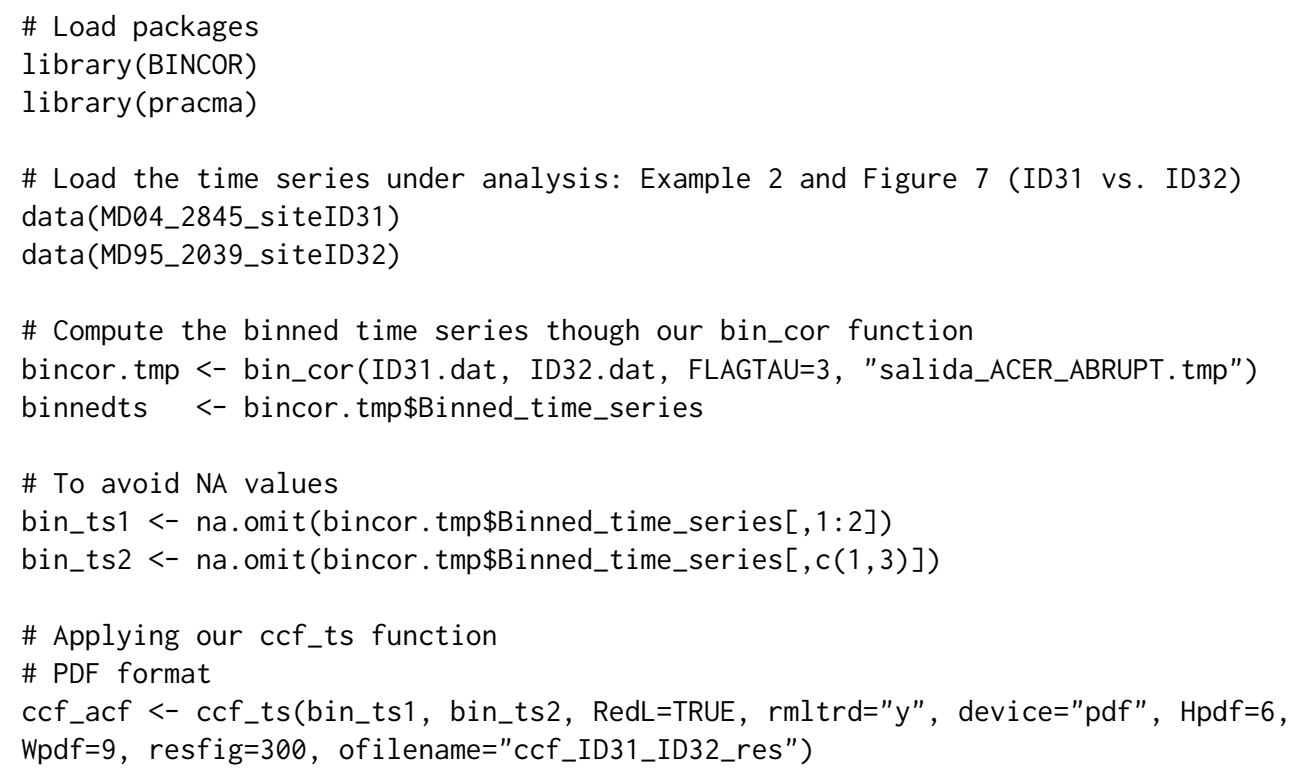




\section{Summary}

We present a computational package named BINCOR (BINned CORrelation) that can be used to estimate the correlation between two unevenly spaced climate time series which are not necessarily sampled at identical points in time, and between two evenly spaced time series which are not on the same time grid. BINCOR is based on a novel estimation approach proposed by Mudelsee (2010). This statistical technique requires the concept of nonzero persistence times, thus enabling mixing information to be recovered, even when the two timescales examined differ (Mudelsee, 2014). The package contains four functions (bin_cor, cor_ts, ccf_ts and plot_ts) with a number of parameters to obtain a high degree of flexibility in the analysis. BINCOR is programmed in R language and is available from the CRAN repository. The results when BINCOR $\mathrm{s}$ applied to real climate data sets suggest that the R package BINCOR performs and works properly in detecting relationships between instrumental and paleoclimate records.

\section{Acknowledgements}

JMPM was funded by a Basque Government post-doctoral fellowship. MM's work was supported by the European Commission via Marie Curie Initial Training Network LINC (project number 289447) under the Seventh Framework Programme. Thanks to Charo Sánchez for help to use the i2BASQUE HPC facilities, to the two anonymous reviewers and Editor (Olivia Lau) for their input and comments that have improved the quality of the manuscript. The authors thank the support of the computing infrastructure of the i2BASQUE (Basque Government) academic network. The persistence time estimation software is freely available via http://www. climate-risk-analysis. com/software/.

\section{Bibliography}

M. Blaauw. Out of tune: The dangers of aligning proxy archives. Quaternary Science Reviews, 36:38-49, 2012. URL https://doi.org/10.1016/j.quascirev.2010.11.012. [p11]

H. W. Borchers. pracma: Practical Numerical Math Functions, 2015. URL http: //CRAN. R-project. org/ package=pracma. R package version 1.8.8. [p5]

P. Brohan, J. J. Kennedy, I. Harris, S. F. Tett, and P. D. Jones. Uncertainty estimates in regional and global observed temperature changes: A new data set from 1850. Journal of Geophysical Research: Atmospheres, 111(D12), 2006. URL http://dx.doi.org/10.1029/2005JD006548. [p6,7,8]

A. Bunn, M. Korpela, F. Biondi, F. Campelo, P. Merian, F. Qeadan, C. Zang, A. Buras, J. Cecile, M. Mudelsee, and M. Schulz. Dendrochronology Program Library in R, 2015. URL http: //CRAN. Rproject. org/package=dplR. R package version 1.6.3. [p5]

W. Dansgaard, S. Johnsen, H. Clausen, D. Dahl-Jensen, N. Gundestrup, C. Hammer, C. Hvidberg, J. Steffensen, A. Sveinbjornsdottir, J. Jouzel, and G. Bond. Evidence for general instability of past climate from a 250-kyr ice-core record. Nature, 364(6434):218-220, 1993. URL http: //dx. doi .org/ 10.1038/364218a0. [p9]

J. Emile-Geay. Data Analysis in the Earth E Environmental Sciences. Ed. Figshare, 2016. [p1]

D. B. Enfield and D. A. Mayer. Tropical Atlantic sea surface temperature variability and its relation to El Nino-Southern Oscillation. Journal of Geophysical Research: Oceans, 102(C1):929-945, 1997. URL http://dx.doi.org/10.1029/96JC03296. [p8]

E. Frentzos, K. Gratsias, and Y. Theodoridis. Index-based most similar trajectory search. In 2007 IEEE 23rd International Conference Data Engineering, pages 816-825, 2007. URL http://dx. doi .org/10. 1109/ICDE. 2007.367927. [p7]

J. Garcia-Serrano, C. Cassou, H. Douville, A. Giannini, and F. J. Doblas-Reyes. Revisiting the ENSO teleconnection to the Tropical North Atlantic. Journal of Climate, 30(17):6945-6957, 2017. URL https://doi.org/10.1175/JCLI-D-16-0641.1. [p8]

D. L. Gilman, F. J. Fuglister, and J. M. Mitchell Jr. On the power spectrum of "red noise". Journal of the Atmospheric Sciences, 20(2):182-184, 1963. URL https: //doi org/10.1175/1520-0469 (1963) $020<0182:$ OTPSON>2.0. CO;2. [p1]

L. Horowitz. The effects of spline interpolation on power spectral density. IEEE Transactions on Acoustics, Speech, and Signal Processing, 22(1):22-27, 1974. URL http://dx. doi .org/10.1109/TASSP. 1974.1162536. [p1] 
J. Hu, J. Emile-Geay, and J. Partin. Correlation-based interpretations of paleoclimate data-where statistics meet past climates. Earth and Planetary Science Letters, 459:362-371, 2017. URL https: //doi.org/10.1016/j.eps1.2016.11.048. [p11]

M. E. Mann and J. M. Lees. Robust estimation of background noise and signal detection in climatic time series. Climatic Change, 33(3):409-445, 1996. URL https://doi .org/10.1007/BF00142586. [p1]

M. E. Mann, Z. Zhang, S. Rutherford, R. S. Bradley, M. K. Hughes, D. Shindell, C. Ammann, G. Faluvegi, and F. Ni. Global signatures and dynamical origins of the Little Ice Age and Medieval Climate Anomaly. Science, 326(5957):1256-1260, 2009. URL https://doi . org/10.1126/science. 1177303. $[\mathrm{p} 6,7,8]$

U. Mori, A. Mendiburu, and J. Lozano. TSdist: Distance measures for time series data. $R$ package version 3.4, 2, 2015. URL http: //CRAN. R-project. org/package=TSdist. [p7]

U. Mori, A. Mendiburu, and J. A. Lozano. Distance measures for time series in R: The TSdist package. R Journal, 8(2):451-459, 2016. [p7]

M. Mudelsee. TAUEST: A Computer Program for Estimating Persistence in Unevenly Spaced Weather/Climate Time Series. Computers E Geosciences, 28(1):69-72, 2002. URL https://doi. org/10.1016/S0098-3004(01)00041-3. [p1, 2, 5]

M. Mudelsee. Estimating Pearson's correlation coefficient with bootstrap confidence interval from serially dependent time series. Mathematical Geology, 35(6):651-665, 2003. URL https: //doi .org/ 10.1023/B:MATG.0000002982.52104.02. [p1]

M. Mudelsee. Climate Time Series Analysis: Classical Statistical and Bootstrap Methods. Springer-Verlag, 2010. ISBN 9048194814. [p1, 2, 3, 12]

M. Mudelsee. Climate Time Series Analysis: Classical Statistical and Bootstrap Methods. Springer-Verlag, Second edition, 2014. ISBN 9048194814. [p1, 2, 3, 5, 12]

K. Olafsdottir and M. Mudelsee. More accurate, calibrated bootstrap confidence intervals for estimating the correlation between two time series. Mathematical Geosciences, 46(4):411-427, 2014. URL https: //doi.org/10.1007/s11004-014-9523-4. [p1]

K. Rehfeld and G. Bedartha. NESTOOLBOX - Toolbox for the Analysis of Non-Equidistantly Sampled Time Series, 2014. URL http://tocsy. pik-potsdam. de/nest. php. Matlab/Octave, version 1.01. [p1]

K. Rehfeld and J. Kurths. Similarity estimators for irregular and age-uncertain time series. Climate of the Past, 10(1):107-122, 2014. URL https://doi .org/10.5194/cp-10-107-2014. [p1]

K. Rehfeld, N. Marwan, J. Heitzig, and J. Kurths. Comparison of correlation analysis techniques for irregularly sampled time series. Nonlinear Processes in Geophysics, 18(3):389-404, 2011. URL https://doi.org/10.5194/npg-18-389-2011. [p1]

J. Roberts, M. Curran, S. Poynter, A. Moy, T. van Ommen, T. Vance, C. Tozer, F. S. Graham, D. A. Young, C. Plummer, J. Pedro, D. Blankenship, and M. Siegert. Correlation confidence limits for unevenly sampled data. Computers $\mathcal{E}$ Geosciences, 104:120-124, 2017. URL https://doi . org/10. 1016/j. cageo.2016.09.011. [p1]

P. Robinson. Estimation of a time series model from unequally spaced data. Stochastic Processes and their Applications, 6(1):9-24, 1977. URL https://doi .org/10. 1016/0304-4149(77)90013-8. [p1,2]

K. Roucoux, L. De Abreu, N. Shackleton, and P. Tzedakis. The response of NW Iberian vegetation to North Atlantic climate oscillations during the last 65 kyr. Quaternary Science Reviews, 24(14): 1637-1653, 2005. URL https://doi.org/10.1016/j.quascirev.2004.08.022. [p9,11]

M. F. Sanchez Goni, A. Landais, W. J. Fletcher, F. Naughton, S. Desprat, and J. Duprat. Contrasting impacts of Dansgaard-Oeschger events over a western European latitudinal transect modulated by orbital parameters. Quaternary Science Reviews, 27(11):1136-1151, 2008. URL https://doi . org/10. 1016/j.quascirev.2008.03.003. [p9]

G. P. Weedon. Time-Series Analysis and Cyclostratigraphy: Examining Stratigraphic Records of Environmental Cycles. Cambridge Univ Press, Cambridge, 2003. [p1]

D. S. Wilks. Statistical Methods in the Atmospheric Sciences, volume 100. Academic press, 2011. [p1] 
E. W. Wolff, S. P. Harrison, R. Knutti, M. F. Sanchez Goni, O. Wild, A.-L. Daniau, V. Masson-Delmotte, I. C. Prentice, and R. Spahni. How has climate responded to natural perturbations? In S. E. Cornell, I. C. Prentice, J. I. House, and C. J. Downy, editors, Understanding the Earth System: Global Change Science for Application, pages 72-101. Cambridge University Press, 2012. [p9]

Josue M. Polanco-Martinez

Basque Centre for Climate Change - BC3

Sede Building 1, 1st floor - Scientific Campus of the UPV/EHU

48940 Leioa

$\mathcal{E}$

Econometrics Research Group - Institute of Public Economics

University of the Basque Country

48015 Bilbao

SPAIN

josue.m.polanco@gmail.com, josue.polanco@bc3research.org

Martin A. Medina-Elizalde

Dept. of Geosciences, Auburn University

2050 Beard Eaves Coliseum, 36849 Auburn, AL

USA

mam0199@auburn.edu

Maria F. Sanchez Goni

Ecole Pratique des Hautes Etudes (EPHE), PSL University \& UMR EPOC CNRS 5805, University of Bordeaux Allee Geoffroy St Hilair, 33615 Pessac

FRANCE

maria.sanchez-goni@u-bordeaux.fr

Manfred Mudelsee

Climate Risk Analysis, 37581 Bad Gandersheim

$\mathcal{E}$

Alfred Wegener Institute (AWI) - Helmholtz Centre for Polar and Marine Research

27570 Bremerhaven

GERMANY

mudelsee@climate-risk-analysis.com 